Original Article

\title{
Age-related changes in the tactile pressure sensitivity threshold in the sole of the foot in diabetic patients
}

\author{
Kazuki Kimura, RPT, PhD ${ }^{1 *}$, Yoshiaki Endo, RPT, MS ${ }^{2)}$ \\ 1) Department of Rehabilitation, Niigata University of Rehabilitation: 2-16 Uenoyama, Murakami, \\ Niigata 958-0053, Japan \\ 2) Department of Rehabilitation, Shioya Hospital, International University of Health and Welfare, \\ Japan
}

\begin{abstract}
Purpose] Patients diagnosed with diabetes mellitus (DM) are known to experience sensory disturbances in their feet secondary to diabetic neuropathy (DN). The sole of the foot tactile pressure sensitivity threshold in patients with DM was evaluated in addition to examining age-related effects based on the sites studied. Whether the tactile pressure sensitivity threshold in the sole of the foot in elderly patients with DM was associated with the duration of DM or aging was evaluated. [Participants and Methods] Our study included 204 patients with DM (136 males and 68 females). Patients were divided into the following age groups: 20-39 (20 patients), 40-49 (26 patients), 50-59 (34 patients), 60-69 (60 patients), 70-79 (45 patients), and 80-89 years (19 patients). Using SemmesWeinstein monofilament testing, the tactile pressure sensitivity threshold at the sole of the foot was evaluated in the bilateral halluces, first metatarsal head, fifth toe metatarsal head, and the heels, and age-related effects were examined. [Results] At all the sites that were examined, we observed an age-related increase in the tactile pressure sensitivity threshold value, and this finding demonstrated a positive partial correlation with the duration of DM. [Conclusion] Aging influences DN-induced degeneration of peripheral nerves. Key words: Diabetes mellitus, The sole of the foot tactile pressure sensitivity threshold, Aging
\end{abstract}

(This article was submitted Feb. 16, 2018, and was accepted Apr. 21, 2018)

\section{INTRODUCTION}

Diabetic neuropathy (DN) and lower extremity circulatory disturbance are considered to cause diabetes mellitus (DM)related foot lesions ${ }^{1)}$. The sole, which comes into contact with the ground while walking, is prone to the formation of callus and ulcer because of a foot deformity or an abnormality in sole pressure distribution. Because DM-related food lesion is difficult to treat, it poses a high risk of amputation of the lower extremities, which is 15-40 times higher in patients with DM than in healthy individuals ${ }^{2}$. Notably, a lower limb amputation of a patient with DM increases the burden on the stump, wound, and opposite lower extremity, leading to recurrence of ulcer and re-amputation. Consequently, there is a risk of development of further decline in physical function. Hence, periodic foot examination and risk assessment of foot lesions are essential for the prevention of the onset and recurrence of DM-related foot lesions.

Based on the examination of the tactile pressure sensitivity threshold using the Semmes-Weinstein monofilament (SWM) for the risk assessment of DM-related foot lesion, when a patient does not respond to a thickness of 5.07 (tactile pressure sensitivity, $10 \mathrm{~g}$ ), the suspicion of $\mathrm{DN}^{3}$ and risk of amputation of a lower limb due to DM-related ulcer significantly increases ${ }^{4}$. Hence, the American Diabetes Association mandates a tactile pressure sensitivity threshold by a filament of size 5.07 when evaluating the risk of $\mathrm{DN}^{5)}$.

*Corresponding author. Kazuki Kimura (E-mail: 11s1039@g.iuhw.ac.jp)

(C2018 The Society of Physical Therapy Science. Published by IPEC Inc.

(c) (1) $($ This is an open-access article distributed under the terms of the Creative Commons Attribution Non-Commercial No Derivatives CC. ${ }_{\text {BY }}$ ND (by-nc-nd) License. (CC-BY-NC-ND 4.0: https://creativecommons.org/licenses/by-nc-nd/4.0/) 
A report by the Organization for Economic Cooperation and Development (OECD) revealed that the lifespan of humans has prolonged worldwide ${ }^{6}$, creating a new problem of aging in patients with DM. The purpose of this research was to examine the sole of the foot tactile pressure sensitivity threshold of elderly DM patients and determine whether it is related with DM duration or aging.

\section{PARTICIPANTS AND METHODS}

The participants of this study included 204 patients with DM (136 males and 68 females) who underwent physical therapy during educational hospitalization for DM. All participants were divided into the following age groups: 20-39, 40-49, 50-59, 60-69, 70-79, and 80-89 years. All patients requiring dialysis and those with severe DM complications, such as leg amputation, were excluded from this study. This cross-sectional study compared groups for each age range. We obtained informed consent from all the participants. Furthermore, this study was approved by the Institutional Review Board of International University of Health and Welfare (Ohtawara, Tochigi, Japan; approval number: 14-Io-72).

Evaluation of the sole of the foot tactile pressure sensitivity threshold included the use of SWM (Sakai Medical Co., Ltd., Tokyo, Japan) to evaluate the sole of the foot tactile pressure sensitivity threshold using six sizes $(2.83,3.61,4.31$, 4.56, 5.07, and 6.65). The pressure was applied for approximately $1.5 \mathrm{~s}$ until attaining a bent at a right angle of the bilateral forefeet (halluces, first metatarsal head, and fifth toe metatarsal head) and heel (total eight sites), and the correct size was considered as the representative value of each site. The stage at which a correct response could be obtained for size 2.83 (i.e., the thinnest filament) was set as stage 1; a seven-stage scale was used to evaluate the correct response for size 6.65 as stage 6 and nonresponse for size 6.65 as stage 7.

We compared the gender ratio of each group using the $\chi^{2}$ test, and other essential information was assessed using the oneway analysis of variance. The sole of the foot tactile pressure sensitivity threshold was evaluated by comparing age-related effects according to the site. First, the correlation between age and the sole of the foot tactile pressure sensitivity threshold was evaluated using the Spearman's partial rank correlation coefficient. In addition, DM duration was set as a control variable to eliminate the effect of the duration of DM. The correlation between DM duration and the sole of the foot tactile pressure sensitivity threshold was evaluated using the Spearman's rank correlation coefficient. Next, we used the Friedman test for the comparison of sites according to the age group and Bonferroni's method for multiple comparisons in this study. However, a 70 year-old patient's disease duration was unknown, and one patient was excluded from the correlation analysis. Furthermore, we considered $\mathrm{p}<0.05$ as statistically significant for all tests. The statistical software SPSS $21.0 \mathrm{~J}$ (IBM SPSS Japan, Inc.) was used for statistical processing.

\section{RESULTS}

Table 1 summarizes the primary data results of this study. We observed no differences in gender ratios between the groups. Higher age ranges correlated with longer disease duration, smaller stature, and decreased body weight. In addition, the body mass index of the younger-aged groups was higher than that of the older-aged groups.

Table 2 shows the number of individuals (ratio) with the sole of the foot tactile pressure sensitivity threshold according to the site for each age group. No patient in this study responded at size 6.65. In addition, the median sole of the foot tactile pressure sensitivity threshold at all sites revealed a higher value with increasing age.

The results of the Spearman's partial correlation analysis adjusted with DM duration revealed that the sole of the foot tactile pressure sensitivity threshold of all sites had a significant positive partial correlation with age $(\mathrm{p}<0.05)$ : halluces, $\rho=0.220$; first metatarsal heads, $\rho=0.251$; fifth toe metatarsal heads, $\rho=0.224$; and heels, $\rho=0.291$. The results of the Spearman's correlation analysis revealed that the sole of the foot tactile pressure sensitivity threshold of all sites had a significant positive correlation with DM duration ( $\mathrm{p}<0.05)$ : halluces, $\rho=0.188$; first metatarsal heads, $\rho=0.144$; fifth toe metatarsal heads, $\rho=0.139$; and heels, $\rho=0.122$.

The sole of the foot tactile pressure sensitivity threshold of the heel at all ages was significantly higher than that of the forefoot. In addition, no differences were observed in the sole of the foot tactile pressure sensitivity threshold among the four sites.

\section{DISCUSSION}

An investigation of the age-related changes of the sole of the foot tactile pressure sensitivity threshold in patients with DM using the SWM revealed that the sole of the foot tactile pressure sensitivity threshold exhibited a high value as the age increased.

A prolonged DM duration could result in the recurrence of hyperglycemia and the progression of DN. The results of the Spearman's partial correlation coefficient adjusted by the DM duration revealed that the sole of the foot tactile pressure sensitivity threshold of all sites had a significant positive partial correlation with age $(\rho=0.220-0.291)$. Aging showed highly positive correlation with the sole of the foot tactile pressure sensitivity threshold compared with that by DM duration. Regarding DP, it was suggested to DM duration that in addition and aging itself affected elderly DM patient's sole tactile 


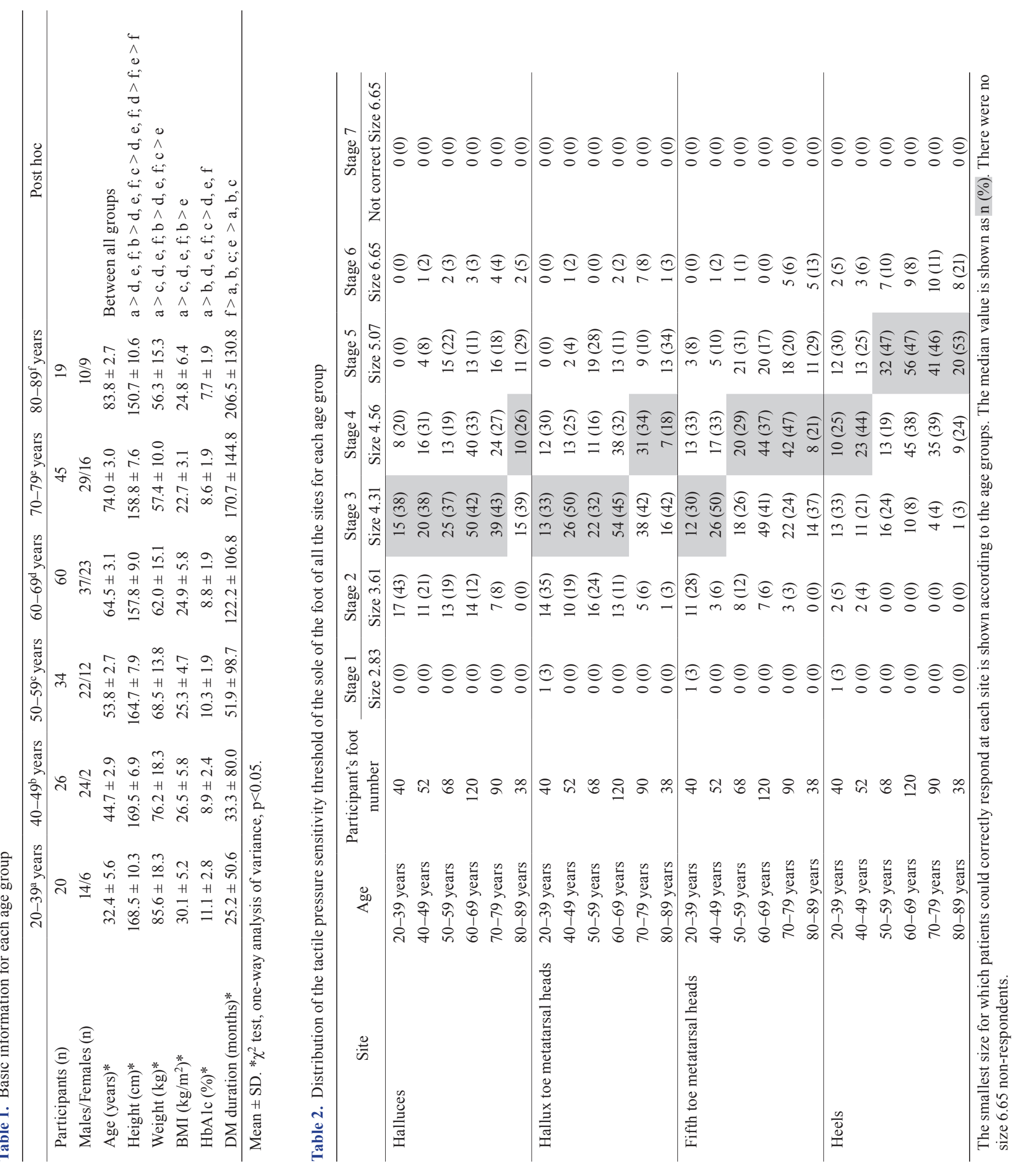


pressure sensitivity threshold. In contrast, we observed no differences in gender in all age groups; in the absence of gender differences regarding the severity of $\mathrm{DN}$, it appeared that differences because of gender were small ${ }^{7)}$. In addition, $\mathrm{HbA} 1 \mathrm{c}$, an indicator of the mean blood glucose level in the past 1-2 months, was high (mean: 11.1\%) in the 20-39 age group. Hence, it is suggested that when young persons who are in school or working undergo educational hospitalization of DM, they must take a leave of absence from school or work. Hence, it can be concluded that understanding of the examination and treatment for DM was not adequate. The effluvium of a myelin sheath and the tabes of a spermatic filament by DN affected the denature of the peripheral nerve. In addition, it was considered that the neurodegeneration of the aging arose ${ }^{8)}$. As the increased the sole of the foot tactile pressure sensitivity threshold is a risk factor for a foot lesion, the evaluation of the feet in elderly patients with DM is imperative for the prevention of foot lesions.

In this study, the sole of the foot tactile pressure sensitivity threshold of the heel was significantly higher than that in the forefoot in patients with DM of all age groups. Most cases of DN are classified as diabetic polyneuropathy (DP), and because of the gradual disturbance of proximal regions from the more distal regions of the bilateral extremities, the forefoot of patients with $\mathrm{DM}$ is significantly affected by $\mathrm{DN}^{9)}$. Hence, the results of this study suggest that $\mathrm{DP}$ affected patients with $\mathrm{DM}$ up to the heel. In addition to motor and sensory neuropathy, DP could be attributed to autonomic dysfunction ${ }^{10)}$, which, in turn, could lead to decline in sweating of the sole and, thus, drying of the skin causing failure of the barrier function. Furthermore, the results suggest that the thickening of the skin advanced because of the load applied to the heel when the heel is in contact with the ground during walking. Thus, in the heels of patients with DM, the sole of the foot tactile pressure sensitivity threshold was seemingly higher than that of the forefoot because of the influence of DN and autonomic nerves. When evaluating the sole of the foot tactile pressure sensitivity threshold using the SWM, it is essential to evaluate not only the forefoot affected by DN but also the extent of effects of DN. Finally, this study suggests the need to evaluate the sole of the foot tactile pressure sensitivity threshold of the entire sole. The degeneration of the peripheral nerves by DN was influenced by aging. Moreover, it was suggested that aging and not DM duration affected DM patient's sole tactile pressure sensitivity threshold. It was suggested that the aging has affected an old-age DM patient's sole tactile pressure sensitivity threshold. This study had some limitations. First, only age-related changes in patients with DM are discussed here because complications of DM and modes of transportation were not investigated. In the future, it is necessary to mention lifestyle factors (e.g., complications, lower extremity circulation, and smoking) in addition to age.

\section{Conflict of interest}

None.

\section{REFERENCES}

1) Moulik PK, Mtonga R, Gill GV: Amputation and mortality in new-onset diabetic foot ulcers stratified by etiology. Diabetes Care, 2003, 26: 491-494. [Medline] [CrossRef]

2) Frykberg RG, Zgonis T, Armstrong DG, et al. American College of Foot and Ankle Surgeons: Diabetic foot disorders. A clinical practice guideline (2006 revision). J Foot Ankle Surg, 2006, 45: S1-S66. [Medline] [CrossRef]

3) Feng Y, Schlösser FJ, Sumpio BE: The Semmes Weinstein monofilament examination as a screening tool for diabetic peripheral neuropathy. J Vasc Surg, 2009, 50: 675-682, 682.e1. [Medline] [CrossRef]

4) Nather A, Bee CS, Huak CY, et al.: Epidemiology of diabetic foot problems and predictive factors for limb loss. J Diabetes Complications, 2008 , 22 : 77-82. [Medline] [CrossRef]

5) American Diabetes Association: (9) Microvascular complications and foot care. Diabetes Care, 2015, 38: S58-S66. [Medline] [CrossRef]

6) OECD iLibrary: Life expectancy at birth, 1970 and 2013 (or nearest years). http://www.keepeek.com/Digital-Asset-Management/oecd/social-issues-migration-health/health-at-a-glance-2015/life-expectancy-at-birth-1970-and-2013-or-nearest-years_health_glance-2015-graph15-en\#.WhVdfM8Uljo (Accessed Nov. 22, 2017).

7) Bansal D, Gudala K, Muthyala H, et al.: Prevalence and risk factors of development of peripheral diabetic neuropathy in type 2 diabetes mellitus in a tertiary care setting. J Diabetes Investig, 2014, 5: 714-721. [Medline] [CrossRef]

8) Dyck PJ, Giannini C: Pathologic alterations in the diabetic neuropathies of humans: a review. J Neuropathol Exp Neurol, 1996, 55: 1181-1193. [Medline] [CrossRef]

9) Baraz S, Zarea K, Shahbazian HB, et al.: Comparison of the accuracy of monofilament testing at various points of feet in peripheral diabetic neuropathy screening. J Diabetes Metab Disord, 2014, 13: 19. [Medline] [CrossRef]

10) Tesfaye S, Boulton AJ, Dyck PJ, et al. Toronto Diabetic Neuropathy Expert Group: Diabetic neuropathies: update on definitions, diagnostic criteria, estimation of severity, and treatments. Diabetes Care, 2010, 33: 2285-2293. [Medline] [CrossRef] 